\title{
Error Analysis of Approximate Calculation of Voltage Divider Biased Common-Emitter Amplifier
}

\author{
Xinwu Chen, Jingjing Xue, Shuangbo Xie, Wenxia Huang, Peng Wang, Ke Gong, Lijuan Zhong \\ School of Physics and Electronic Engineering, Xinyang Normal University, Xinyang, China \\ Email: chenxinwu@126.com
}

How to cite this paper: Chen, X.W., Xue, J.J., Xie, S.B., Huang, W.X., Wang, P., Gong, K. and Zhong, L.J. (2017) Error Analysis of Approximate Calculation of Voltage Divider Biased Common-Emitter Amplifier. Circuits and Systems, 8, 247-252. https://doi.org/10.4236/cs.2017.810017

Received: July 15, 2017

Accepted: October 14, 2017

Published: October 17, 2017

Copyright $\odot 2017$ by authors and Scientific Research Publishing Inc. This work is licensed under the Creative Commons Attribution International License (CC BY 4.0). http://creativecommons.org/licenses/by/4.0/

\begin{abstract}
Voltage divider biasing common emitter amplifier is one of the core contents in analog circuit curriculum, and almost all of traditional textbooks apply approximate calculation method to estimate all characteristic parameters. In calculating quiescent point, transistor base current is generally ignored to get the approximate base potential and emitter current, then other operating parameters, and AC small signal parameters can be acquired. The main purpose of this paper is to compare traditional and Thevenin equivalent methods and to get the difference of the two methods. A Formula is given to calculate the error of the traditional method. Example calculating reveals that the traditional method can generate an error about $10 \%$, and even severe for small signal amplifier with higher quiescent point.
\end{abstract}

\section{Keywords}

Common-Emitter Amplifier, Quiescent Point, Thevenin Equivalent Circuit, Small Signal Amplifier

\section{Background}

An amplifier is one of the most important contents of electronic circuit systems. The main reason is that almost all the analog signals from the sensors are very weak and could not drive loads directly. The main function of the amplifiers is amplifying the weak signals so that the signal can become strong enough for practical applications. How to improve the characteristics of amplifiers is always one ongoing problem. Lots of scholars have carried out a lot of work from different directions.

We can classify the research into two different fields. The first region is the 
study of new material devices. In this region, H. C. Lin and G. M. Rebeiz [1], K. Ma, T. B. Kumar and K. S. Yeo [2] carried out their work focusing on new silicon-germanium alloy material devices and made great progress. H. C. Lin and G. M. Rebeiz aim at how to improve the frequency characteristics and power gain. While K. Ma, T. B. Kumar, and K. S. Yeo studied the reconstructing approaches. The second region is built on common and general problems [3] [4] [5]. In this field, Z. Yang, D. N. Nath, Y. Zhang proposed a special common-emitter amplifier which can amplify voltage and current at same time. R. S. Assaad and J. Silva-Martinez did a research about graph approach for amplifiers. While S. H. Hsu, W. P. Kang and J. L. Davidson described the nature of vacuum microelectronic integrated differential amplifier. We can put this paper in the second region as we talked above.

As shown in Figure 1, the voltage divider-based common-emitter amplifier is most popular in traditional textbooks and practical applications [6] [7]. As we know, we can design or analyze the circuit from quiescent point to AC small-signal situation which means that we divide the problem into two steps. In the first step, that is to say, during the quiescent point determination, we can deal with the circuit in two most common different ways. The first method is that we can use an approximate approach. In the approach, we ignore the current of base called $I_{B}$ because in reality the base current is very small compared with the biased current through the two biasing resistors in almost all the cases. The second method is based on Thevenin equivalent methods. In this process, we do not ignore the base current and we can get accurate values of several voltages and currents. As we all know, no matter which method is used, after we get the values of currents and voltages of quiescent point, we can determine the working region of the bipolar junction transistor. Generally speaking, the transistor should be biased in the active forward region which means that we can use the constraint $I_{C}=\beta I_{B}$, and the voltage between collector and emitter $V_{C E}$ should be greater than $V_{C E S}$ which stands for the critical saturation voltage and for small power transistors, we often think that $V_{C E S}$ should be about $0.2 \mathrm{~V}-0.3 \mathrm{~V}$.

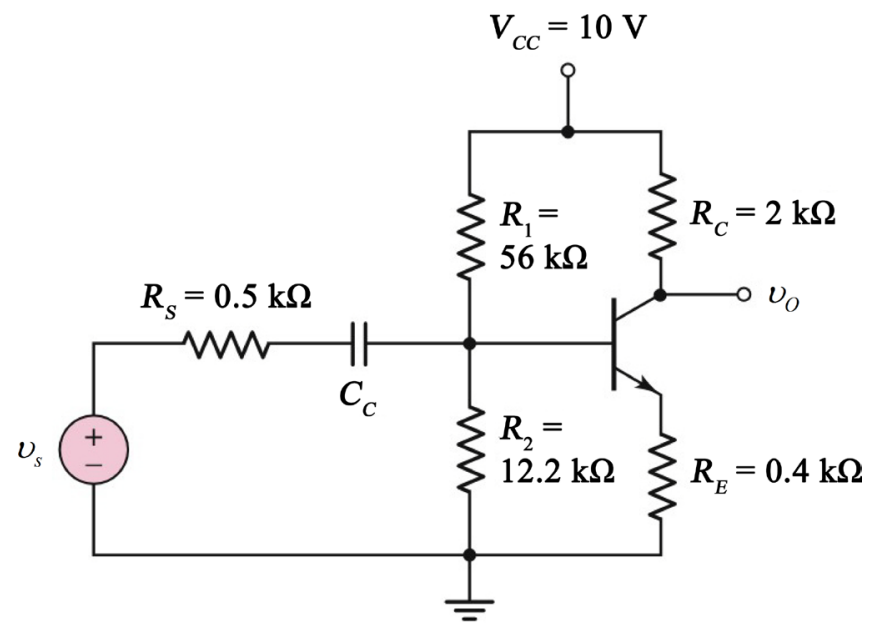

Figure 1. Voltage divider biasing common emitter amplifier. 
If we can determine that the transistor is biased in active forward region, we can continue the design or analysis of dynamic situation using hybrid $\Pi$ equivalent circuit.

As we mentioned above, we can use different methods to calculate the quiescent point currents and voltages. Then, three questions are standing in front of us, they are:

1) If we ignore the base current as we described in method 1, what is the significant level of the effect to the quiescent point currents and voltages?

2) How can we get a formula so that we evaluate the error range of the first method?

3) If there is a relation between the error of the quiescent point currents and voltages and the quiescent point currents and voltages?

In this paper, we will give the answers for the three questions above.

\section{DC Analyses Using First Approach}

For the integrity of this paper, let us give a short brief review for the first approach to evaluate the quiescent point currents and voltages.

For the circuit shown in Figure 1, assuming that the biasing current here we call $I_{1}$ which flows through $R_{1}$ and $R_{2}$ is far greater than the base current IB, that is, $I_{1} \gg \mathrm{I}_{\mathrm{B}}$, hence we think that the base current IB is essentially zero. So the base potential can be evaluated as Equation (1). Collector and emitter currents can be evaluated as shown in Equation (2) and be denoted as $I_{C}$ and $I_{E}$, respectively.

The voltage between collector and emitter $V_{C E}$ can be calculated as shown in Equation (3). Base current IB can be evaluated as in Equation (4).

$$
\begin{gathered}
V_{B} \approx \frac{R_{2}}{R_{1}+R_{2}} \cdot V_{C C} \\
I_{C} \approx I_{E}=\frac{V_{B}-V_{B E}}{R_{E}} \\
V_{C E}=V_{C C}-I_{C} R_{C}-I_{E} R_{E} \approx V_{C C}-I_{C}\left(R_{C}+R_{E}\right) \\
I_{B}=\frac{I_{E}}{1+\beta}=\frac{V_{B}-V_{B E}}{R_{E}(1+\beta)}
\end{gathered}
$$

\section{Thevenin Equivalent Methods to Quiescent Point}

If we do not ignore the base current $\mathrm{I}_{B}$, we can use Thevenin equivalent methods to find quiescent point currents and voltages. The basic equivalent circuit is shown in Figure 2. Equivalent voltage source $V_{T H}$ and Equivalent resistance $R_{T H}$ can be calculated according to Equations ((5) and (6)), respectively. The quiescent current IBQ can be acquired from Equation (7) and the result of IBQ can be expressed as Equation (8).

$$
\begin{gathered}
V_{T H}=\frac{R_{2}}{R_{1}+R_{2}} V_{C C} \\
R_{T H}=R_{1} / / R_{2}
\end{gathered}
$$




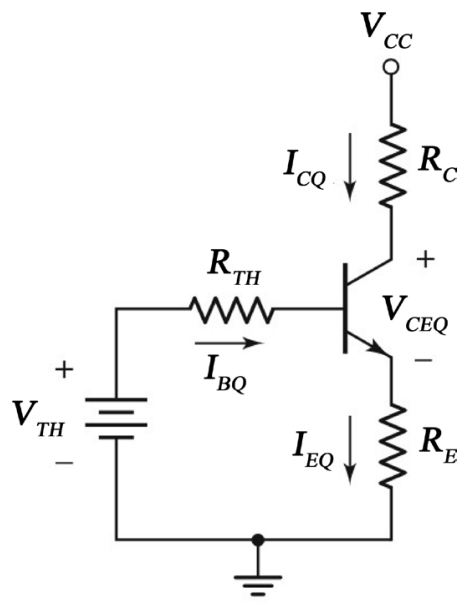

Figure 2. Thevenin equivalent circuit of Figure 1.

$$
\begin{gathered}
V_{T H}=I_{B Q} R_{T H}+V_{B E}+I_{E Q} R_{E}=I_{B Q} R_{T H}+V_{B E}+(1+\beta) I_{B Q} R_{E} \\
I_{B Q}=\frac{V_{T H}-V_{B E}}{R_{T H}+(1+\beta) R_{E}}
\end{gathered}
$$

\section{Comparisons of the Two Approaches}

By comparing the quiescent point current $I_{B}$ and $I_{B Q}$ according to Equations ((4) and (8)), we can get the absolute error $I_{B Q_{-} E r r}$ of $I_{B}$ due to the approximate calculating as shown in Equation (9).

$$
I_{B Q_{-} E r r}=I_{B Q}-I_{B}=\frac{V_{T H}-V_{B E}}{R_{T H}+(1+\beta) R_{E}}-\frac{V_{B}-V_{B E}}{(1+\beta) R_{E}}
$$

Notice that $V_{T H}-V_{B E}$ equals to $V_{B}-V_{B E}$, hence $I_{B Q_{-} E r r}$ can be written as Equation (10)

$$
I_{B Q_{-} E r r}=\left(V_{T H}-V_{B E}\right)\left(\frac{1}{R_{T H}+(1+\beta) R_{E}}-\frac{1}{(1+\beta) R_{E}}\right)
$$

So we can get the relative error of quiescent point current IB from the first method as shown in Equation (11)

$$
\text { Error }=\frac{I_{B Q_{-} E r r}}{I_{B Q}}=\left|\frac{\frac{1}{R_{T H}+(1+\beta) R_{E}}-\frac{1}{(1+\beta) R_{E}}}{\frac{1}{R_{T H}+(1+\beta) R_{E}}}\right|=\frac{R_{T H}}{(1+\beta) R_{E}}
$$

From Equation (11) we can see that bigger $R_{T H}$ means bigger error which is caused by first method. According to the parameters in Figure 1, and if we assume the common-emitter hybrid parameter $\beta=100$, we can get the relative error defined in Equation (11) is about 25\%. It should be noted that the error is not small. And if we use the parameters in literature [6], which means $\beta=60$, $V_{C C}=10 \mathrm{~V}, R_{1}=60 \mathrm{~K} \Omega, R_{2}=20 \mathrm{~K} \Omega, R_{C}=3 \mathrm{~K} \Omega, R_{E}=2 \mathrm{~K} \Omega$, the value of error is 14.1\%. We listed the detail results in Table 1 . 
Table 1. Quiescent point parameters acquired by the two methods.

\begin{tabular}{cccccccc}
\hline & & $I_{B}(\mu \mathrm{A})$ & $\mathrm{I}_{\mathrm{B}}$ error & $I_{C}(\mathrm{~mA})$ & $I_{C}$ error & $V_{C E}(\mathrm{~V})$ & $V_{C E}$ error \\
\hline $\begin{array}{c}\text { Circuit from } \\
\text { [7] }\end{array}$ & Method 1 & 27.0 & $5.4 \mu \mathrm{A}$ & 2.70 & $0.54 \mathrm{~mA}$ & 3.52 & $1.29 \mathrm{~V}$ \\
& Method 2 & 21.6 & $25 \%$ & 2.16 & $25 \%$ & 4.81 & $26.8 \%$ \\
$\begin{array}{c}\text { Circuit from } \\
\text { [6] }\end{array}$ & Method 1 & 27.5 & $3.4 \mu \mathrm{A}$ & 1.65 & $0.2 \mathrm{~mA}$ & 7.75 & $0.9 \mathrm{~V}$ \\
& Method 2 & 24.1 & $14.1 \%$ & 1.45 & $14.1 \%$ & 8.65 & $10.4 \%$
\end{tabular}

Table 2. Relative errors of quiescent point IBQ under different $R_{2}$ of Figure 1 .

\begin{tabular}{ccccccc}
\hline $\mathrm{R}_{2}(\mathrm{~K} \Omega)$ & 5 & 6 & 7 & 8 & 9 & 10 \\
$\mathrm{I}_{\mathrm{BQ}}(\mu \mathrm{A})$ & 2.66 & 5.84 & 8.82 & 11.60 & 14.22 & 16.67 \\
$\mathrm{I}_{\mathrm{B}}(\mu \mathrm{A})$ & 2.96 & 6.63 & 10.18 & 13.61 & 16.95 & 20.18 \\
Error $(\%)$ & 11.36 & 13.41 & 15.40 & 17.33 & 19.19 & 21.00 \\
$\mathrm{R}_{2}(\mathrm{~K} \Omega)$ & 11 & 12 & 13 & 14 & 15 & 16 \\
$\mathrm{I}_{\mathrm{BQ}}(\mu \mathrm{A})$ & 18.99 & 21.17 & 23.24 & 25.19 & 27.05 & 28.81 \\
$\mathrm{I}_{\mathrm{B}}(\mu \mathrm{A})$ & 23.31 & 26.35 & 29.31 & 32.18 & 34.97 & 37.68 \\
Error $(\%)$ & 22.76 & 24.46 & 26.12 & 27.72 & 29.28 & 30.80 \\
\hline
\end{tabular}

From the results as shown in the two examples above and Equation (11), if we want the relative error of $I_{B}$ lower, we need lower resistance ratio between $R_{T H}$ and $(1+\beta) R_{E}$. In other words, the biasing resistances should be smaller. As we all know, smaller biasing resistance means higher biasing current which cause higher DC power consumption. Furthermore, it can decrease the input resistance and hence the source voltage amplification factor will be decreased. This kind of effects causes lower performance of amplifiers.

In order to further reveal the relation between the value of relative error of $I_{B Q}$ and quiescent point $I_{B Q}$, we keep the resistance of $R_{1}$ and sweep the resistance of $R_{2}$ from $5 \mathrm{~K} \Omega$ to $16 \mathrm{~K} \Omega$, get the value of $\mathrm{I}_{\mathrm{B}}$ from first method and $I_{B Q}$ from the second method, and the relative error as shown in Table 2.

From Table 2 we can see that with the increasing of resistance of $R_{2}, I_{B}$ and $I_{B Q}$ increase at the same time, the relative error of $I_{B Q}$ also increases; this rule can be described by Equation (11). Because the increase of $R_{2}$ cause the increase of $R_{T H}$ while $(1+\beta) R_{E}$ keeps constant. In practical applications, for small signal amplifier circuit, we hope the power consumption of the biasing circuit lower hence the resistance $R_{T H}$ is often high. It means that the relative error of first method can be not very high; the value is often higher than $10 \%$.

It should be noted that the reason which causes the error is the ignorance of the current through the biasing resistors in the first method. When the resistance called $R_{T H}$ increases, the current $I_{B Q}$ will decays. On the other hand, the increasing of the degradation resistor called $R_{E}$ can make the error of $I_{B Q}$ stable because of feedback effect.

\section{Conclusion}

In this paper, we compared the two common-used methods to the solution of 
voltage-divider common-emitter amplifier, giving the formula of the difference of the quiescent point base current. From the formula and some simulation results we can know that the higher quiescent point means more significant error of quiescent base current. The contribution of this paper can improve the understood feature of this theory and avoid significant errors in determining the quiescent of bipolar junction transistor amplifiers and furthermore, help the designers to character the features of amplifiers for the AC analysis. We will discuss some more complex circuits for the two methods studied in this paper in the future to avoid errors in the design process.

\section{Acknowledgements}

This work is supported by the Project of Education Department of Henan Province (12A510020), 2015 National College Students' Innovative Entrepreneurial Training Program from Department of China (12129), the Science and Technology Project of Henan Province (172102210455), and Project of Graduate Student Research Innovation Fund from Xinyang Normal University (2016KYJJ35).

\section{References}

[1] Lin, H.C. and Rebeiz, G.M. (2016) A 70-80-GHz SiGe Amplifier with Peak Output Power of $27.3 \mathrm{dBm}$. IEEE Transactions on Microwave Theory and Techniques, 64, 2039-2049. https://doi.org/10.1109/TMTT.2016.2574863

[2] Ma, K., Kumar, T.B. and Yeo, K.S. (2015) A Reconfigurable K-/Ka-Band Power Amplifier with High PAE in $0.18-\mu \mathrm{m}$ SiGe BiCMOS for Multi-Band Applications. IEEE Transactions on Microwave Theory and Techniques, 63, 4395-4405. https://doi.org/10.1109/TMTT.2015.2495129

[3] Yang, Z., Nath, D.N., Zhang, Y.J., Khurgin, B. and Rajan, S. (2015) Common Emitter Current and Voltage Gain in III-Nitride Tunneling Hot Electron Transistors. IEEE Electron Device Letters, 36, 436-438. https://doi.org/10.1109/LED.2015.2413934

[4] Assaad, R.S. and Silva-Martinez, J. (2009) A Graphical Approach to Teaching Amplifier Design at the Undergraduate Level. IEEE Transactions on Education, 52, 39-45. https://doi.org/10.1109/TE.2008.917190

[5] Hsu, S.H., Kang, W.P., Davidson, J.L., Huang, J.H. and Kerns, D.V. (2012) Vacuum Microelectronic Integrated Differential Amplifier. Electronics Letters, 48, 1219-1220. https://doi.org/10.1049/el.2012.1245

[6] Kang, H. (2001) Analog Part in Fundamental of Electronic Technology. 4th Edition, High Education Publishing House, Beijing.

[7] Donald, N. (2007) Microelectronics Circuit Analysis and Design. 3rd Edition, Tsinghua University Publishing House, Beijing. 